\title{
Tratamento de linfoma do manto com Rituximab
}

\author{
Treatment of mantle-cell lymphoma with Rituximab
}

\author{
Jane de Almeida Dobbin,' Henry Luiz Najman² e Maria Inez Pordeus Gadelha
}

o linfoma da célula do manto (Classificação Real) corresponde a uma neoplasia de célula $B$ madura, periférica (Classificação WH O ), ao linfoma centrocítico, centroblástico, subtipo centrocitóide (Classificação de Kiel) e ao linfoma difuso de células pequenas, clivadas - grau intermediário (Working Formulation). Às suas características morfológicas somam-se a translocação $\mathrm{t}(11: 14) ;$ bcl-1, rearranjo do gene ciclina D 1, expressão forte SIgM e IgD, CD 5 positivo, CD 20 positivo, CD 23 negativo, CD 10 negativo, CD 43 positivo e CD 103 negativo.

Eleé considerado uma doença do homem idoso, cujas manifestações clínicas são linfadenomegalia generalizada, algumas vezes esplenomegalia maciça, manifestação em sangue periférico e predileção pelo acometimento do trato gastrintestinal por lesões polipoídes difusas. Setenta por cento dos pacientes são diagnosticados em estádio IV. Na maioria dos trabalhos publicados, observou-se sobrevida média global de 3 anos e sobrevida livre de doença de 1 ano, com poucos sobreviventes de longo tempo.

Em relação ao tratamento, o maior problema está em se atingir a remissão completa do linfoma. $0 \mathrm{~s}$ esquemas de quimioterapia combinadamaisfreqüentemente utilizados são o CVP (ciclofosfamida, vincristina e prednisona) e $\mathrm{O} \mathrm{CHOP}$ (ciclofosfamida, doxorrubicina, vincristina e prednisona). 0 percentual de resposta terapêutica varia de $60 \%$ a $80 \%$, sendo que a resposta completa se dá em $30 \%$ a $60 \%$ dos casos. U m estudo que comparou a efetividade do CVP com o CHOP não demonstrou diferença na sobrevida global ( $84 \%$ vs. $88 \%$ ) nem na sobrevida livre de falha terapêutica ( $41 \%$ vs. $58 \%$ ) dos doentes. Esquemas mais agressivos têm sido testados, como o hyperCVAD, que resultou em sobrevida livre de doença em 3 anos superior àquela obtida com CHOP $(72 \%$ vs. $28 \%)$. Por sua vez, poliquimioterapia com Fludarabina, $M$ itoxantrona e D exametasona (FND), associando-se ou não a ciclofosfamida, tem sido testada em pacientes com linfomas de baixo grau responsivos à quimioterapia, cujos resultados são promissores.

D evido aos resultados de sobrevida de longo tempo serem pobres, pacientes jovens e saudáveis têm sido submetidos a transplante de medula óssea, autólogo ou alogenéico. Os seus resultados ainda não são conclusivos, apesar de alguns pacientes apresentarem sobrevida maior, quando submetidos a esses procedimentos.

Em relação ao Rituximab, trata-se de um anticorpo monoclonal quimérico anti-CD 20, cuja ação antilinfomatosa é, provavelmente, devida a toxicidade celular intermediada por complemento e anticorpo, inibindo a proliferação celular e induzindo a apoptose.

Estudos clínicos têm avaliado a resposta ao tratamento com Rituximab, tanto noscasos

${ }^{1} \mathrm{M}$ édica, Chefe do Serviço de H ematologia do H ospital do Câncer I, Instituto N acional de Câncer, Rio de Janeiro, RJ - Brasil.

${ }^{2} \mathrm{M}$ édico, Instituto N acional deC âncer, Rio de Janeiro, RJ - Brasil 
de linfomas não H odgkin indolentes como nos de linfomas não $\mathrm{H}$ odgkin mais agressivos. O s estudos clínicos iniciais que levaram à aprovação deste medicamento pelo FDA incluíram pacientes com recaída de linfomas não Hodgkin de baixo grau e foliculares, utilizando estemedicamento deforma isolada. Posteriormente, o Rituximab foi avaliado como tratamento inicial nos linfomas não Hodgkin indolentes.

$\mathrm{H}$ ainsworth et $\mathrm{al}^{1}$ avaliaram 62 doentes de Linfoma Folicular (LF) e Leucemia Linfocítica Crônica (LLC), forma leucêmica do linfoma linfocítico bem diferenciado, não tratados previamente, obtendo-se $47 \%$ de resposta objetiva, com $7 \%$ de resposta completa e $40 \%$ de resposta parcial, sendo que $48 \%$ dos pacientes ficaram com doença estável. Resultados semelhantes em 50 doentes de LF e LLC foram observados por Gutheil et al. ${ }^{2} \mathrm{Um}$ terceiro estudo, publicado por Colombat et al, ${ }^{3}$ avaliou 50 doentes de LF e anti-CD 20 positivo. Após 78 dias de tratamento, observou-se resposta global de $73 \%$, com $33 \%$ de resposta completa e $47 \%$ de resposta parcial.

Foran et al ${ }^{4}$ avaliaram, em estudo de Fase II, o uso de Rituximab para pacientes com linfoma da célula do manto, imunocitoma, linfoma linfocítico de células $B$ pequenas. Dos 120 pacientes incluídos, observou-se resposta completa em $30 \%$ deles. Os percentuais de resposta completa por tipo histológico foram os seguintes: linfoma da célula do manto sem tratamento prévio: $38 \%$; linfoma da célula do manto previamente tratado: $37 \%$; Imunocitoma: $28 \%$ e linfoma linfocítico de células B pequenas: $14 \%$. Somente $10 \%$ dos doentes com linfoma da célula do manto alcançaram resposta completa.. Como conclusão deste estudo, 0 Rituximab tem, como agente único, atividade moderada sobre o linfoma da célula do manto e Imunocitoma e atividade mínima sobre 0 linfoma linfocítico de células $B$ pequenas. 0 mesmo Foran, em outro trabalho publicado com outros colaboradores, ${ }^{5}$ concluiu que 0 Rituximab atua contra o linfoma de célula do manto e pode induzir resposta completa em uma minoria dos doentes; e que o tratamento prévio com agentes alquilantes associa-se a respostas globais significantemente mais baixas.

McLaughlin et al ${ }^{6}$ avaliaram o uso de Rituximab em 166 pacientes com linfoma de células B. 0 percentual de resposta foi $48 \%$, com seguimento médio de 11,8 meses. $A$ conclusão deste estudo é que a ação do Rituximab é semelhante a da quimioterapia com agente único. A conclusão deste estudo foi que são necessários outros estudos para garantir a indicação desse medicamento, inclusive devendo ser testado em conjunto com quimioterapia padrão.

Coiffier et al ${ }^{7}$ avaliaram, em estudo de Fase II, o uso de Rituximab em 54 pacientes com linfoma não H odgkin agressivo, recidivado ou refratário à quimioterapia - pacientes com linfoma difuso de grandes células, linfoma da célula do manto, ou outros linfomas de célula $B$ de grau intermediário e agressivo. D ezessete pacientes responderam, sendo que 5 deles al cançaram resposta completa e 12, resposta parcial. $\mathrm{N}$ a análise de intenção de tratamento (definida para identificar fatores associados com um prognóstico favorável), o percentual de resposta foi de $31 \%$. Entre 12 pacientes com diagnóstico de linfoma da célula do manto, 4 (33\%) apresentaram resposta. A conclusão do estudo foi que o Rituximab tem atividade significativa no linfoma difuso de grandes células e linfoma da célula do manto, devendo ser testado em combinação com quimioterapia, em pacientes com estes diagnósticos.

$M$ aloney et $a^{8}$ avaliaram, também em estudo de Fase II, o uso de Rituximab em 37 pacientes com linfoma não $\mathrm{H}$ odgkin de baixo grau. Resposta foi observada em 17 pacientes (46\%). Como 3 pacientes foram excluídos, 34 pacientes foram analisados, e, entre estes, 3 pacientes ( $9 \%$ ) tiveram resposta completa e 14 (41\%), resposta parcial. Concluiu-se que o Rituximab deverá ser testado em outros estudos em pacientes com outros tipos de linfoma não H odgkin, seja de forma isolada ou em poliquimioterapia.

Esquemas quimioterápicos combinados com Rituximab, para linfoma não H odgkin de baixo grau, foram posteriormente testados e avaliados. C zuczman et $\mathrm{al}^{9}$ publicaram 0 primeiro estudo com Rituximab combinado com CHOP, que incluiu 38 pacientes com linfoma não Hodgkin de baixo grau, cuja 
maioria não havia sido anteriormente tratada. O bteve-se $100 \%$ de resposta, sendo que completa em $55 \%$ dos casos. O utras combinações foram testadas, como Rituximab eFN D, sem diferença de respostas em torno dos 12 meses pós-tratamento e sem vantagens quando comparado aos esquemas tradicionais, como CHOP e CVP;10 e Rituximab e Fludarabina, ainda com dados muito recentes para assegurar-se 0 impacto sobre a sobrevida global, tempo em que se dá a falha terapêutica, e sobrevida livre de doença. ${ }^{11}$

$\mathrm{H}$ oward et al ${ }^{12}$ testaram a eficácia do Rituximab associado ao $\mathrm{CHOP}$ em 40 doentes de linfoma da célula do manto, em estádio II a IV e não previamente tratados. Foi observada resposta completa (resposta completa e sem confirmação molecular) em $48 \%$ e resposta parcial, também em $48 \%$ deles. Foi observado um período livre de doença de 16,6 meses; entretanto, 28 de 40 pacientes ainda tiveram recaída ou progressão do linfoma.

Como se pode observar, em relação ao uso Rituximab no linfoma não $\mathrm{H}$ odgkin de baixo grau e no linfoma da célula do manto existem vários estudos clínicos publicados, porém todos estudos de Fase II (análise de resposta e toxicidade), inexistindo estudo de Fase III (análise comparativa de resultados entre dois ou mais esquemas terapêuticos) publicado até o momento.

Em relação aos linfomas agressivos, os estudos clínicos sugerem que o Rituximab pode ser útil no seu tratamento. Coiffier et al ${ }^{13}$ realizaram um estudo de Fase III que incluiu 399 doentes idosos (com mais de 60 anos de idade) com linfoma difuso de células $B$ grandes, e encontraram que ela aumenta 0 percentual de resposta completa e prolonga a sobrevida livre de doença e a sobrevida global, sem aumento significativo da toxicidade. Existe um outro estudo do Eastern Cooperative 0 ncology $G$ roup (ECO G), em andamento, avaliando CH OP vs. CHOP e Rituximab. ${ }^{14}$

Cheson, ${ }^{15}$ do Instituto $\mathrm{N}$ acional de Câncer dos Estados Unidos, teceu considerações muito importantes sobre 0 tratamento com 0 esquema $\mathrm{CHOP}$ e Rituximab: primeira - "All these exciting re sults initiated the current trend to adopt
CHOP plus Rituximab as the new standard of care for patients with B-cell lymphoma. However, this approach is premature." Analisando 0 estudo de Coiffier, ${ }^{13}$ ele alerta que, apesar deste estudo ter demonstrado percentual de sobrevida superior com a associação de CHOP e Rituximab (70\%) comparando-se com CH O P isolado (57\%), para um seguimento mediano de 2 anos, as diferenças na curva de sobrevida começam a diminuir aos 2,5 anos de seguimento e continuarão a diminuir com morte dos pacientes devidos a idade, complicações do tratamento e complicações relacionadas à idade. Seguimentos de maior tempo são necessários para confirmar se esta vantagem do esquema CHOP e Rituximab irá se manter. Ele ainda enfatiza que a eficácia de tratamento para um tipo de linfoma, não é necessariamente benéfico para outros tipos de linfoma; e volta a enfatizar que o esquema C H O P é o tratamento padrão para o linfoma difuso de grandes células, mas não para o linfoma folicular e outros linfomas não $\mathrm{H}$ odgkin de baixo grau. O utras combinações com Rituximab têm demonstrado resultados promissores, porém estudos clínicos randomizados são necessários para definir 0 melhor esquema de tratamento. Segunda "W hat is the harm in routinely adding Rituximab to chemotherapy for nonH odgkin's lymphoma?" Ele lembra que o tratamento com Rituximab por 4 semanas custa aproximadamente 12 mil dólares; que a pergunta se a quimioterapia combinada com Rituximab é melhor do que quimioterapia seguida de Rituximab para linfomas não Hodgkin indolentes permanece não respondida; e como também permanece sem resposta a questão referente aos efeitos adversos fatais relacionados ao $\mathrm{CHOP}$ e Rituximab.

Armitage et $a^{16}$ tecem 0 seguinte comentário, em relação ao Rituximab: "Further trials will be necessary to define the place of Rituximab in the management of patients with mantle cell lymphoma." Por sua vez, $M$ aloney e M ocharnuk, ${ }^{17}$ bem como $\mathrm{H}$ orning e M ocharnuk, ${ }^{14}$ no levantamento feito sobre a terapia com anticorpos monoclonais de doenças de linfócitos $B$, inclusive neoplasias malignas, chamam a atenção para a 
necessidade de continuidade das experiências relatadas.

$\mathrm{N}$ esse mesmo trabalho, $\mathrm{H}$ orning e M ocharnuk, ${ }^{14}$ na parte referente a transplante de medula óssea, e sabendo-se já que os linfomas indolentes não são completamente controlados por quimioterapia em altas doses seguida de transplante autólogo de medula óssea, visto que se reinfundem células linfomatosas persistentes na cultura tratada (in vitro), buscaram atualizar os estudos sobre se anticorpos monoclonais, como 0 Rituximab, possam manter os percentuais de resposta, as células imunocompetentes e 0 número de células tronco, se usado in vivo. A partir dos resultados de 12 estudos, eles ressaltam o quanto estes resultados foram instigantes, a ponto de dois estudos de Fase III, envolvendo quimioterapia em altas doses e transplante autólogo de medula óssea randomizando-se os doentes em grupos com e sem Rituximab, pré e pós-transplante. U m desses estudos é europeu, com início dado em setembro de 2001; e o outro, estadunidense, previsto para iniciar-se em 2002.

Especificamente quanto ao trabalho de $M$ agni et al, ${ }^{18}$ publicado em 2000, foram incluídos 25 doentes, sendo 10 deles (dos quais três com linfoma de célula do manto) no grupo de quimioterapia em altas doses seguida de transplante autólogo de medula óssea e 15 doentes (dos quais sete com linfoma de célula do manto) no outro grupo, que também recebeu Rituximab, além dessas mesmas quimioterapia e transplante. Restrito à análise das condições técnicas específicas do transplante e das respostas terapêuticas obtidas, mas não à de sobrevida dos doentes, este trabalho concluiu que, paralelamente à obtenção de células tronco sem linfoma residual (PCR negativo), a quimio-imunoterapia produziu remissão completa, clínica e molecular, em todos os 14 pacientes elegíveis, entre os quais se incluíram 6 com linfoma de célula do manto. Estetrabal ho mostra também que o purging da medula in vivo é factível e possivelmente superior às técnicas in vitro disponíveis àquele ano.

Já $M$ angel et al, ${ }^{19}$ em um estudo de Fase II , também utilizando C H O P, quimioterapia em altas doses, transplante autólogo de medula óssea e Rituximab, iniciado em 1999 e que incluiu 13 doentes, todos com linfoma de célula do manto previamente não tratados, concluíram que, em que pese o pequeno número de doentes e o tempo de seguimento curto, os resultados preliminares são encorajadores e a duração da resposta precisa ser determinada ao longo de um período de observação maior.

Por seu lado, Gianni et al, ${ }^{20}$ com uma amostra de 28 doentes, também concluem que, dos 20 doentes avaliados em termos de resposta molecular, $100 \%$ deles apresentaram células tronco sem linfoma, ao exame de PCR, demonstrando, com isso, que a associação de quimioterapia em altas doses e Rituximab é eficiente para o purging da medula óssea in vivo. Também concluem, embora ainda com um curto período de seguimento, que essa associação pode ter vir a ter seu papel no tratamento dos doentes de linfoma de célula do manto que respondem mal à quimioterapia padrão.

Press, ${ }^{21}$ como participante em recente congresso da American Society of Clinical Oncology, apresentou uma revisão da experiência internacional no tratamento do linfoma de célula do manto com transplante autogênico, alogenéico e sem mieloablação, e discutiu as condutas aplicáveis a subgrupos especiais deste tipo de linfoma, sem que resultados finais pudessem ser estabelecidos. Por sua vez, Cabanillas, ${ }^{22}$ nesse mesmo congresso, analisando os resultados dos diferentes esquemas quimioterápicos para linfoma de célula do manto, ressalta a importânica da doxorrubicina no tratamento deste tumor; a tolerabilidade de doentes idosos ao hiperCVAD alternado com metotrexato e citarabina; e os resultados similares, entre doentes com menos de 66 anos (os elegíveis para transplante de células progenitoras de medula óssea), do hiper-CVAD mais transplante e esquemas associados a Rituximab.

Também, não se pode deixar de salientar o estudo de Sweetenham et al, ${ }^{23}$ no qual chamaram a atenção para a necessidade de se desenvolver estudos prospectivos para confirmação de resultados terapêuticos da associação de CHOP, fludarabina e Rituximab, com o necessário estabelecimento 
da relação entre o benefício e o custo dessa associação.

Face ao exposto, verifica-se que o uso de Rituximab para o tratamento de linfomas não $\mathrm{H}$ odgkin ainda não pode ser considerado um procedimento estabelecido, standard. E, especificamente para o linfoma de célula do manto, persiste como um procedimento experimental. Por isso, estudos investigacionais devem continuar sendo desenvolvidos, tanto com doentes de linfomas de baixo grau como de linfomas agressivos, com a intenção de aumentar, nesses doentes, os percentuais de resposta completa (molecular e clínica) e da sobrevida livre de linfoma.

\section{REFERÊNCIAS BIBLIOGRÁFICAS}

1. H ainsworth JD, BurrisH A III, M orrissey LH . Rituximab as first-line and maintenance therapy for patients with indolent nonH odgkin'slymphoma. Proc Am Soc Clin O ncol 2001;(Abstr 79):20a.

2. Gutheil JC, FunicaneD, Rodríguez, R. Phase II study of Rituximab in patients with previously untreated low-grade or follicular nonH odgkin'slymphoma. Proc Am Soc Clin O ncol 2000;12(Abstr 79):22a.

3. Colombat P, Salles G, BrousseN, Eftekhari P, Soubeyran $P$, D elwail V, et al. R ituximab (anti CD-20 monoclonal antibody) assinglefirst line therapy for patients with follicular lymphoma with a low tumor burden: clinical and molecular evaluation. Blood 2001;97:101-6.

4. Foran JM, Rohatiner $A Z$, C unnigham $D$, Popescu RA, Solal-C eligny P, G hielmini M , et al. European phasell study of rituximab (chimeric anti-CD 20 monoclonal antibody) for patients with newly diagnosed mantlecell lymphoma and previously treated mantlecell lymphoma, immunocytoma, and small B-cell lymphocytic Iymphoma. J Clin O ncol 2000;18(2):317-24.

5. Foran JM, C unningham $D, C$ oiffier $B$, SolalCeligny P, Reyes F, G uielmini M , et al. Treatment of mantlecell lymphoma with Rituximab (chimeric monoclonal anti-CD 20 antibody): analysis of factors associated with response. Ann Oncol 2000;11(Suppl 1):117-21.

6. McLaughlin P, G rillo-LópezAJ, Link BK, Levy $R$, Czuczman MS, Williams $M E$, et al. Rituximab chimeric anti-CD 20 monoclonal antibody therapy for relapsed indolent lym- phoma: half of patients respond to a four-dose treatment program. J Clin O ncol 1998;16(8):2825-33.

7. Coiffier $B, H$ aioun $C$, Ketterer $N$, Engert $A$, Tilly $H$, Johnson $P$, et al. Rituximab (antiCD 20 monoclonal antibody) for thetreatment of patients with relapsing or refractory aggressive lymphoma: a multicenter phasell study. Blood 1998;92(6):1927-32.

8. M aloney D G, Grillo-LópezAJ, WhiteCA, Bodkin $D$, Schilder RJ, N eidhart JA, et al. ID EC C2B 8 (Rituximab) anti-CD 20 antibody therapy in patients with relapsed low-grade non-Hodgkin's lymphoma. Blood 1997;90(6):2188-95.

9. Czuczman MS, Grillo-López AJ, W hite CA, Saleh M , G ordon L, Lo Buglio AF, et al. Treatment of patients with low-grade B-cell lymphoma with thecombination of chimeric antiCD 20 monoclonal antibody and $\mathrm{CH} O \mathrm{P}$ che motherapy. J Clin 0 ncol 1999;17(1):268-76.

10. $C$ abanillas $F, M$ CLaughlin $P, H$ agemeister $F$. M olecular responses with FND + Rituxan chemoimmunotherapy for stage IV indolent follicular non-H odgkin's lymphoma. Blood 2000;97(Abstr 3504):331a.

11. Czuczman MS, Fallon A, M ohr A. Phase II study of rituximab plusfludarabinein patients with low-gradelymphoma: final report. Blood 2001;98(Abstr 2518):601a.

12. H oward OM, Gribben JG, N euberg DS, Grossbard M, Poor C, Janicek MJ, et al. Rituximab and $\mathrm{CH} O \mathrm{O}$ induction therapy for newly diagnosed mantle-cell lymphoma: molecular completeresponsesarenot predictive of progression-free survival. J Clin O ncol 2002;20(5):1288-94.

13. Coiffier $B$, Lepage $E$, Briére J, $H$ erbrecht $R$, Tilly H , Bouabdallah R, et al. CH O P Chemotherapy plus rituximab compared with $\mathrm{CH} \mathrm{OP}$ alone in elderly patients with diffuse large- $\mathrm{B}$ cell lymphoma. N Engl J Med 2002; 346(4):235-42.

14. H orning SJ , M ocharnuk RS. M onoclonal antibody therapy in b-cell-related disordersand malignancies: clinical upadate. Available from: www.medscapecom/viewprogram/1420_index.

15. Cheson BD . CH O P plusR ituximab: balancing facts and opinion. N Engl J Med 2002;346(4):280-2.

16. Armitage $\mathrm{O}, \mathrm{M}$ auch $\mathrm{PM}, \mathrm{H}$ arris $\mathrm{NL}$, Bierman P. N on-H odgkin'slymphomas. In: D eVitaVT $J \mathrm{r}$, H ellman S, RosenbergAS. Cancer: principles and practice of oncology. Philadelphia: 
Lippincott Williams and Wilkins; 2001. p. 2256-316.

17. Maloney DG, M ocharnuk RS. O ptimizing anti-CD 20 immunotherapy of lymphomas and chronic lymphocytic leukemia: medical education collaborative. Available from: www.medscape.com/viewprogram/627_index.

18. M agni M , Di N icola M , D evizzi L, M atteucci $P$, Lombardi F, $G$ andola $L$, et al. Succesfful in vivo purging of $C D$ 34-containing peripheral blood harvests in mantlecell and indolent lymphoma: evidence for a role of both chemotherapy and rituximab infusion. Blood 2000;96(3):864-9.

19. $M$ angel J, Buckstein $R$, Imrie $K$, Spaner $D$, Crump M , TompkinsK, et al. Immunotherapy with Rituximab following high-dose therapy and autologous stem-cell transplantation for mantle cell Iymphoma. Semin 0 ncol
2002;29(1 Suppl 2):36-59.

20. G ianni AM , Cortelazzo S, M agni M , M artelli M . Rituximab: enhancing stem cell transplantation in mantlecell lymphoma. BoneM arrow Transplant 2002;29(Suppl 1):S10-3.

21. Press OW. Treatment of mantlecell lymphoma: stem-cell transplantation, radioimmunotherapy, and management of mantle-cell lymphoma subsets. Philadelphia: American Society of Clinical O ncology; 2002. p. 407-15.

22. C abanillasF. M anagement of mantlecell lymphoma. Philadelphia: American Society of Clinical O ncology; 2002. p. 416-19.

23. Sweetenham J, H iekeK, Kerrigan M , H oward P, Smartt PF, M cl ntyre, et al. Cost-minimization analysis of $\mathrm{CHOP}$, fludarabine and rituximab for thetreatment of relapsed indolent B-cell non-H odgkin's lymphoma in the U.K. BrJ H aematol 1999; (106):47-54. 